\title{
ETHNO-MEDICINAL USES OF WILD HERBS AND SHRUBS OF TEHSIL YAZMAN, PUNJAB, PAKISTAN
}

\author{
Iram Fatima*, Maria Munawar, Sana Iqbal and Zunaira Sadaf \\ Government Sadiq College Women University, Bahawalpur, Pakistan \\ *Corresponding author's e-mail: iramfatima46@yahoo.com
}

\begin{abstract}
The present study was conducted to document the ethno-medicinal uses of wild herbs and shrubs of Tehsil Yazman, District Bahawalpur, Punjab. Information was gathered by interviewing local people. The collected plants were identified with the help of Flora of Pakistan. Plants were preserved and mounted on herbarium sheets and placed in the Department of Botany, Government Sadiq College Women University, Bahawalpur, Pakistan. A total of 118 species belonging to 35 families were identified. It was noticed that the indigenous people of the study area use plants in different ways in their daily life such as medicines, fuel, fruit, vegetable, forage/fodder, ornamental etc. Three families were found in abundance i.e. Poaceae, Fabaceae and Asteraceae. To the best of our knowledge, it is the first report on the ethno-medicinal uses of wild herbs and shrubs of Tehsil Yazman. It will be helpful in providing a baseline for future studies relating to pharmacological as well as biochemical studies of these wild plant species.
\end{abstract}

Keywords: Ethnobotany, biodiversity, medicinal plants, ethno-medicines, phytochemicals.

\section{INTRODUCTION}

Total area of Pakistan is $80,943 \mathrm{~km}^{2}$, it comprises of unique biodiversity including about 6,000 plant species (Ali and Qaiser, 1986). Yazman tehsil is sub-division of Bahawalpur District in the Punjab province of Pakistan. Its original name (with diacritics) was Yazman Mandi. The human population comprises of different races, i.e. Kutwal, Sheikh, Makhy, Mahar, Bhain, Bohar, Jam, Arain and Tanwari (Ahmad et al., 2012).

Ethnobotany is the study of relationship between people and plants, and documentation of indigenous knowledge for conservation and sustainable usage of plant resources (Zereen and Khan, 2012). It is an investigation of plants valuable to individuals. Pakistan, like many other countries of the world, is bestowed with rich plant assets utilized for medicinal and also for different purposes (Ali and Qaiser, 1986). The poor people around the world trust medicinally valuable plants for treatment of different ailments. Some species are specific for a particular disease while some species have multiple uses; but, sometimes they have mixed usage (Shinwari et al., 2011). The interest for medicinal plants is rising day by day across the globe because of unsatisfactory performance and high expenses of modern medicines.

The plants have been used as timber to build shelters, firewood to keep places warm, to cook food, as medicine to treat themselves and to graze the livestock. Plants play a dynamic role in our lives mainly because of phytochemicals possessing variety of biological activities (Ajaib et al., 2010). Hence, ethno-medicinal research is very useful in the development of new drugs. Although many ethnobotanical surveys have been conducted by different workers (Qureshi $e t$ al., 2011; Ahmad et al., 2012; Malik et al., 2013; Choudhary et al., 2014; Wariss et al., 2014; Butt et al., 2015) in different areas of Pakistan but to our knowledge no systematic studies on ethno-medicinal potential of wild herbs and shrubs of Tehsil Yazman has been made. Hence, studies were conducted to explore the floristic biodiversity of the area and to record the traditional and medicinal uses of wild herbs and shrubs of the area.

\section{MATERIALS AND METHODS}

Study area: Yazman tehsil is rich in natural plant resources especially wild herbs and shrubs. The mean winter temperature of the area is $6.5^{\circ} \mathrm{C}$ and mean summer temperature is $34-38^{\circ} \mathrm{C}$. Annual rainfall of $100-250 \mathrm{~mm}$, usually occurs in winter and spring whereas subsoil water is brackish (Ahmad et al., 2012). Economic activity depends on favorable climatic and agricultural yield. The vegetation mainly comprises of xeromorphic species, which are adapted to various environmental stresses, such as high salinity, high temperature and low nutrient availability. Local plants are used as ethnobotanical, as well as pharmacological activities by inhabitants.

Ethnobotanical data collection and analysis: Field trips, to collect information about the ethno-medicinal uses of plants by local people in Tehsil Yazman (Pakistan), were conducted during 2016. Plant specimens were collected and identified with the help of The Flora of Pakistan (Nasir and Ali, 2001). Specimens were dried, pressed and mounted on herbarium sheets. All collected specimens were deposited in the 
Government Sadiq College Women University Bahawalpur for future references. Data was grouped according to use, i.e. vegetables, fruits, medicinal, fodder, fuel wood, fencing, hedges and ornamental.

\section{RESULTS}

Total 118 wild species belonging to 35 families were investigated ethno-medicinally during field trips. Poaceae was found to be the largest family with 21 species followed by Fabaceae ( 9 species), Asteraceae ( 8 species $)$ Chenopodiaceae (7 species) and Aizoaceae and Amaranthaceae (6 species each) while Capparidaceae and Solanaceae comprised of 5 species each and rest of the families included 1-4 members. The collected plant species were arranged in alphabetically as per family name. Uses of all the collected plant species along with their parts used were recorded (Table 1).

Table 1. Ethno-medicinal uses of some wild herbs and shrubs of Tehsil Yazman, Pakistan.

\begin{tabular}{|c|c|c|c|c|c|c|c|c|c|}
\hline S. No. & Botanical name & $\begin{array}{l}\text { Growth } \\
\text { Form }\end{array}$ & $\begin{array}{l}\text { Parts } \\
\text { Used }\end{array}$ & Fd & Fw & $\begin{array}{l}\mathbf{F \&} \\
\mathbf{H}\end{array}$ & Orn. & $\begin{array}{l}\text { Fr. \& } \\
\text { Veg. }\end{array}$ & Medicinal Uses \\
\hline \multicolumn{10}{|c|}{ Acanthaceae } \\
\hline 1. & Blepharis scindica & Herb & Sds, rts & $\sqrt{ }$ & $\sqrt{ }$ & & & & General debility, urinary discharge \\
\hline \multicolumn{10}{|c|}{ Aizoaceae } \\
\hline 2. & Gisekia pharnaceoides & Herb & Lvs, sds & $\sqrt{ }$ & & & & $\sqrt{ }$ & Cure asthma and diarrhea, purgative \\
\hline 3. & Limeum indicum & Herb & Ap & $\sqrt{ }$ & $\sqrt{ }$ & & & $\sqrt{ }$ & Treatment of burns \\
\hline 4. & Sesuvium sesuvioides & Herb & Stm,rts & & & & & $\sqrt{ }$ & Small pox, measles, cough, cold \\
\hline 5. & $\begin{array}{l}\text { Trianthema } \\
\text { portulacastrum }\end{array}$ & Herb & $\mathrm{Wp}$ & $\sqrt{ }$ & & & & $\sqrt{ }$ & $\begin{array}{l}\text { Cough, poultice, fungal infections, gonorrhea } \\
\text { and venereal discharge }\end{array}$ \\
\hline 6. & Trianthema triquetra & Herb & Ap & $\sqrt{ }$ & & & & & Ulcer and chronic fever \\
\hline 7. & Zaleya pentandra & Herb & Wp & $\sqrt{ }$ & & & & & Stomach ailment, influenza, kidney stones. \\
\hline \multicolumn{10}{|c|}{ Amaranthaceae } \\
\hline 8. & Achyranthes aspera & Herb & Wp & & $\sqrt{ }$ & & & & $\begin{array}{l}\text { Diuretic, hepatitis, cough and treat asthma, } \\
\text { depression, headache and skin diseases }\end{array}$ \\
\hline 9. & Aerva javanica & Shrub & Wp & $\sqrt{ }$ & $\sqrt{ }$ & $\sqrt{ }$ & $\sqrt{ }$ & & $\begin{array}{l}\text { Cure acne, gall stone and vesicle, Used in } \\
\text { jaundice, urinary trouble and rheumatism and } \\
\text { for headache }\end{array}$ \\
\hline 10. & Amaranthus hybridus & Herb & Ap & $\sqrt{ }$ & & & & $\sqrt{ }$ & Constipation and fever \\
\hline 11. & Amaranthus viridis & Herb & Ap & $\sqrt{ }$ & & & & $\sqrt{ }$ & $\begin{array}{l}\text { Used as emollient and for dysentery and } \\
\text { constipation }\end{array}$ \\
\hline 12. & Digera arvensis & Shrub & Wp & $\sqrt{ }$ & & & & & Diuretic and dyspepsia \\
\hline 13. & Digera muricata & Herb & $\mathrm{Wp}$ & $\sqrt{ }$ & & & $\sqrt{ }$ & $\sqrt{ }$ & Digestive system disorders, urinary disorders \\
\hline \multicolumn{10}{|c|}{ Apiaceae } \\
\hline 14. & Anethum graveolens & Herb & Wp & $\sqrt{ }$ & & & $\sqrt{ }$ & $\sqrt{ }$ & $\begin{array}{l}\text { Diabetes, menstrual cycle, bone development, } \\
\text { stomachic, diuretic, insomnia, cramps, } \\
\text { inflammation of respiratory tract }\end{array}$ \\
\hline 15. & Coriandrum sativum & Herb & Ap & $\sqrt{ }$ & & & $\sqrt{ }$ & $\sqrt{ }$ & $\begin{array}{l}\text { Digestive complaints, small pox, anemia, } \\
\text { fever, measles and cold. }\end{array}$ \\
\hline \multicolumn{10}{|c|}{ Apocyanaceae } \\
\hline 16. & Calotropis procera & Shrub & Wp & & $\sqrt{ }$ & & & & $\begin{array}{l}\text { Fever, rheumatism, indigestion, cold and } \\
\text { diarrhea }\end{array}$ \\
\hline 17. & Carissa carandas & Shrub & Wp & $\sqrt{ }$ & & & $\sqrt{ }$ & $\sqrt{ }$ & $\begin{array}{l}\text { Constipation, diarrhea, mouth ulcer, sore } \\
\text { throat, scabies, epilepsy }\end{array}$ \\
\hline 18. & $\begin{array}{l}\text { Leptadenia } \\
\text { pyrotechnica }\end{array}$ & Shrub & Wp & $\sqrt{ }$ & & & & $\sqrt{ }$ & $\begin{array}{l}\text { Fever, cough, stomach and kidney disorders, } \\
\text { urinary disorders }\end{array}$ \\
\hline \multicolumn{10}{|c|}{ Asphodelaceae (Xanthorracaeae) } \\
\hline 19. & Aloe barbadensis & Herb & Lvs & $\sqrt{ }$ & $\sqrt{ }$ & & $\sqrt{ }$ & $\sqrt{ }$ & $\begin{array}{l}\text { Osteoarthritis, bowel diseases, fever, itching } \\
\text { and inflammation }\end{array}$ \\
\hline 20. & Asphodelus tenuifolius & Herb & Wp & $\sqrt{ }$ & & & & $\sqrt{ }$ & $\begin{array}{l}\text { Diuretic, inflammation, blood purification and } \\
\text { skin diseases }\end{array}$ \\
\hline \multicolumn{10}{|c|}{ Asteraceae (Compositae) } \\
\hline 21. & Cichorium intybus & Herb & Wp & $\sqrt{ }$ & & & & $\sqrt{ }$ & Diarrhea and fever, improving bowel function \\
\hline 22. & Conyza bonariensis & Herb & $\mathrm{Wp}$ & & & & $\sqrt{ }$ & & Constipation, diarrhea \\
\hline 23. & Echinops echinatus & Herb & $\mathrm{Wp}$ & $\sqrt{ }$ & & & & & Liver disorder, jaundice, anorexia, skin itching \\
\hline 24. & Eclipta alba & Herb & Ap & $\sqrt{ }$ & $\sqrt{ }$ & & & & Hair growth and color \\
\hline 25. & Launaea resedifolia & Herb & Lvs & $\sqrt{ }$ & & & & & Hepatic pains, bacterial infections \\
\hline
\end{tabular}




\begin{tabular}{|c|c|c|c|c|c|c|c|c|c|}
\hline S. No. & Botanical name & $\begin{array}{l}\text { Growth } \\
\text { Form }\end{array}$ & $\begin{array}{l}\text { Parts } \\
\text { Used }\end{array}$ & Fd & Fw & $\begin{array}{l}\text { F\& } \\
\text { H }\end{array}$ & Orn. & $\begin{array}{l}\text { Fr. \& } \\
\text { Veg. }\end{array}$ & Medicinal Uses \\
\hline 26. & Sonchus arvensis & Herb & Lvs, rts & $\sqrt{ }$ & & & & & $\begin{array}{l}\text { Asthma, cough, chest infections, } \\
\text { inflammations, kidney pains }\end{array}$ \\
\hline 27. & Sonchus asper & Herb & Lvs, stm & $\sqrt{ }$ & & & & $\sqrt{ }$ & Wound healing, sedative and tonic \\
\hline 28. & Xanthium strumarium & Herb & Lvs & & & & & $\sqrt{ }$ & Fever, nasal and sinus congestion \\
\hline \multicolumn{10}{|c|}{ Boraginaceae } \\
\hline 29. & Heliotropium indicum & Herb & Ap & $\sqrt{ }$ & & & & & $\begin{array}{l}\text { Diuretic, skin problems, inflammations and } \\
\text { tumors }\end{array}$ \\
\hline 30. & Heliotropium strigosum & Herb & $\mathrm{Wp}$ & $\sqrt{ }$ & & & & & $\begin{array}{l}\text { Hepatitis, arthritis, sore eyes, blood } \\
\text { purification, laxative, diuretic, snake bite. }\end{array}$ \\
\hline \multicolumn{10}{|c|}{ Brassicaceae } \\
\hline 31. & Coronopus didymus & Herb & $\mathrm{Wp}$ & $\sqrt{ }$ & & & & & Respiratory disorder and diabetes \\
\hline 32. & Malcolmia africana & Herb & Ap & $\sqrt{ }$ & & & $\sqrt{ }$ & $\sqrt{ }$ & Microbial infections \\
\hline 33. & Sisymbrium irio & Herb & Sds & & & & & $\sqrt{ }$ & $\begin{array}{l}\text { Asthma and eye disease, detoxify liver and } \\
\text { spleen, cough and chest congestion. }\end{array}$ \\
\hline \multicolumn{10}{|c|}{ Capparidaceae } \\
\hline 34. & Capparis decidua & Shrub & Ap & & $\sqrt{ }$ & $\sqrt{ }$ & $\sqrt{ }$ & $\sqrt{ }$ & $\begin{array}{l}\text { Toothache, gum infection, hepatitis, jaundice, } \\
\text { ulcers, bone fraction and to release obesity }\end{array}$ \\
\hline 35 . & Capparis spinose & Herb & $\mathrm{Wp}$ & & & & $\sqrt{ }$ & $\sqrt{ }$ & $\begin{array}{l}\text { Gastro-intestinal infections, liver disorders, } \\
\text { diuretic, cough, anemia }\end{array}$ \\
\hline 36. & Cleome viscosa & Herb & Lvs, sds & & & & & $\sqrt{ }$ & Infections, fever, headache, rheumatism \\
\hline 37. & Dipterygium glaucum & Herb & Ap & $\sqrt{ }$ & & & & & Asthma, expectorant, stimulant \\
\hline 38. & Gynandropsis gynandra & Herb & $\begin{array}{l}\text { Lvs, sds, } \\
\text { rts }\end{array}$ & $\sqrt{ }$ & & & $\sqrt{ }$ & $\sqrt{ }$ & $\begin{array}{l}\text { Gastro-intestinal disease, anemia, scabies, anti- } \\
\text { inflammatory }\end{array}$ \\
\hline \multicolumn{10}{|c|}{ Caryophyllaceae } \\
\hline 39. & Spergula arvensis & Herb & $\mathrm{Wp}$ & $\sqrt{ }$ & & & & & Diuretic \\
\hline 40. & Stellaria media & Herb & $\mathrm{Wp}$ & $\sqrt{ }$ & & & & $\sqrt{ }$ & Cough, kidney pains, constipation \\
\hline \multicolumn{10}{|c|}{ Chenopodiaceae } \\
\hline 41. & Chenopodium album & Herb & Ap & $\sqrt{ }$ & & $\sqrt{ }$ & & $\sqrt{ }$ & $\begin{array}{l}\text { Abdominal pain, tooth decay, throat troubles, } \\
\text { constipation, insect stings and bites, laxative }\end{array}$ \\
\hline 42. & Chenopodium murale & Herb & Ap & $\sqrt{ }$ & & & & $\sqrt{ }$ & Treat ringworms, diuretic and eye diseases \\
\hline 43. & Haloxylon recurvum & Shrub & $\mathrm{Wp}$ & $\sqrt{ }$ & & & & & Intestinal ulcers \\
\hline 44. & Haloxylon salicornicum & Shrub & $\mathrm{Wp}$ & & $\sqrt{ }$ & & & & Dyspepsia, anti-diabetic, anti-bacterial. \\
\hline 45. & Salsola baryosma & Shrub & $\mathrm{Wp}$ & & & & & $\sqrt{ }$ & Skin disease \\
\hline 46. & Salsola imbricate & Shrub & Wp & $\sqrt{ }$ & & & & & Abdominal pain and constipation \\
\hline 47. & Suaeda fruticose & Shrub & $\mathrm{Wp}$ & & & & & & Eye problem, skin disorder \\
\hline \multicolumn{10}{|c|}{ Convolvulaceae } \\
\hline 48. & Convolvulus arvensis & Herb & Lvs & $\sqrt{ }$ & & & $\sqrt{ }$ & & Diuretic, fever, laxative \\
\hline 49. & Cress cretica & Herb & $\mathrm{Wp}$ & & & & $\sqrt{ }$ & & Expectorant, anti-fungal and anti-cancer \\
\hline \multicolumn{10}{|c|}{ Cucurbitaceae } \\
\hline 50. & Citrullus colocynthis & Herb & $\begin{array}{l}\text { Lvs, sds, } \\
\text { rts }\end{array}$ & $\sqrt{ }$ & & & & $\sqrt{ }$ & $\begin{array}{l}\text { Stomach and digestive disorder, diabetes, } \\
\text { constipation, asthma }\end{array}$ \\
\hline 51. & Cucumis melo & Herb & Fr & $\sqrt{ }$ & & $\sqrt{ }$ & $\sqrt{ }$ & $\sqrt{ }$ & Hyperacidity, constipation, anorexia \\
\hline 52. & Mukia maderaspatana & Herb & Lvs, rts & $\sqrt{ }$ & $\sqrt{ }$ & & & & Constipation and gas problems \\
\hline \multicolumn{10}{|c|}{ Cyperaceae } \\
\hline 53. & Cyperus flat & Herb & Ap & $\sqrt{ }$ & & & & & Cure skin disease \\
\hline 54. & Cyperus rotundus & Herb & Wp & $\sqrt{ }$ & & & & $\sqrt{ }$ & $\begin{array}{l}\text { Diarrhea, diabetes, inflammation, malaria, } \\
\text { stomach and bowel disorders }\end{array}$ \\
\hline \multicolumn{10}{|c|}{ Euphorbiaceae } \\
\hline 55. & Euphorbia granulata & Herb & Lvs & $\sqrt{ }$ & & & & & $\begin{array}{l}\text { Dysentery, jaundice, digestive problems, } \\
\text { tumors, cough, asthma }\end{array}$ \\
\hline 56. & Euphorbia hirta & Herb & Ap & $\sqrt{ }$ & $\sqrt{ }$ & & & & $\begin{array}{l}\text { Cough, asthma, dysentery, jaundice, digestive } \\
\text { problems, tumors }\end{array}$ \\
\hline 57. & Euphorbia prostrata & Herb & $\mathrm{Wp}$ & $\sqrt{ }$ & & & & & Hemorrhoids, fever and cure skin problems \\
\hline 58. & Jatropha diocia & Shrub & Ap & $\sqrt{ }$ & & & $\sqrt{ }$ & & Bleeding gums, toothache, laxative \\
\hline \multicolumn{10}{|c|}{ Fabaceae } \\
\hline 59. & Alhagi maurorum & Shrub & $\mathrm{Wp}$ & & & & & $\sqrt{ }$ & $\begin{array}{l}\text { Used to treat piles, migraine, to remove kidney } \\
\text { stones and as a laxative and diuretic }\end{array}$ \\
\hline
\end{tabular}




\begin{tabular}{|c|c|c|c|c|c|c|c|c|c|}
\hline S. No. & Botanical name & $\begin{array}{l}\text { Growth } \\
\text { Form }\end{array}$ & $\begin{array}{l}\text { Parts } \\
\text { Used }\end{array}$ & Fd & $\mathbf{F w}$ & $\begin{array}{l}\mathbf{F \&} \\
\mathbf{H}\end{array}$ & Orn. & $\begin{array}{l}\text { Fr. \& } \\
\text { Veg. }\end{array}$ & Medicinal Uses \\
\hline 60. & Cassia occidentalis & Shrub & Sds, Lvs & $\sqrt{ }$ & & & $\sqrt{ }$ & & $\begin{array}{l}\text { Cough, fever, bronchitis, asthma, liver } \\
\text { complaints, tuberculosis }\end{array}$ \\
\hline 61. & Crotolaria burhia & Shrub & $\mathrm{Wp}$ & $\sqrt{ }$ & & $\sqrt{ }$ & & & Swellings, kidney pains, rheumatism \\
\hline 62. & Lathyrus aphaca & Herb & $\mathrm{Fl}$, sds & $\sqrt{ }$ & & & $\sqrt{ }$ & & Skin infections \\
\hline 63. & Medicago polymorpha & Herb & Ap & $\sqrt{ }$ & & & $\sqrt{ }$ & $\sqrt{ }$ & Constipation, indigestion \\
\hline 64. & Melilotus officinalis & Herb & Wp & $\sqrt{ }$ & & & & $\sqrt{ }$ & Vomiting, headache and to increase appetite \\
\hline 65. & Sesbania sesban & Shrub & Lvs, Sds & $\sqrt{ }$ & $\sqrt{ }$ & & & & $\begin{array}{l}\text { Throat infections, skin infections, cough, cold, } \\
\text { constipation }\end{array}$ \\
\hline 66. & Vicia sativa & Herb & Ap & $\sqrt{ }$ & & & & $\sqrt{ }$ & $\begin{array}{l}\text { Skin infections, asthma, bronchitis, urinary } \\
\text { diseases }\end{array}$ \\
\hline 67. & Vigna radiata & Shrub & Lvs, sds & $\sqrt{ }$ & & & $\sqrt{ }$ & & $\begin{array}{l}\text { Refresh mentality, alleviate heat stroke and } \\
\text { reduce swelling }\end{array}$ \\
\hline \multicolumn{10}{|c|}{ Fumaraceae (Papaveraceae) } \\
\hline 68. & Fumaria indica & Herb & $\mathrm{Wp}$ & $\sqrt{ }$ & & & & & $\begin{array}{l}\text { Fever, influenza, diarrhea, purification of } \\
\text { blood }\end{array}$ \\
\hline \multicolumn{10}{|c|}{ Gentianaceae } \\
\hline 69. & Centaurium pulchellum & Herb & Flw & $\sqrt{ }$ & & & & & $\begin{array}{l}\text { Fever, kidney stones, high blood pressure, } \\
\text { indigestion }\end{array}$ \\
\hline \multicolumn{10}{|c|}{ Malvaceae } \\
\hline 70. & Abutilon indicum & Shrub & $\mathrm{Wp}$ & & $\sqrt{ }$ & & $\sqrt{ }$ & & $\begin{array}{l}\text { Purify the blood, cure cough, asthma and chest } \\
\text { infection, Used as laxative, emollient and } \\
\text { demulcent }\end{array}$ \\
\hline 71. & $\begin{array}{l}\text { Malvastrum } \\
\text { coromendelianum }\end{array}$ & Shrub & $\mathrm{Wp}$ & $\sqrt{ }$ & & & & & $\begin{array}{l}\text { Anti-inflammatory, liver infections, diarrhea, } \\
\text { sore throat, cough }\end{array}$ \\
\hline \multicolumn{10}{|c|}{ Mazaceae } \\
\hline 72. & Mazus pumilus & Herb & $\mathrm{Wp}$ & & & & $\sqrt{ }$ & & Cure typhoid, febrifuge, emmenagogue \\
\hline \multicolumn{10}{|c|}{ Molluginaceae } \\
\hline $\begin{array}{l}73 . \\
\text { Neurac }\end{array}$ & $\begin{array}{l}\text { Mollugo cerviana } \\
\text { daceae }\end{array}$ & Herb & Ap & $\sqrt{ }$ & & & & $\sqrt{ }$ & Laxative, microbial infections \\
\hline $\begin{array}{l}74 . \\
\text { Nyctag }\end{array}$ & $\begin{array}{l}\text { Neurada procumbens } \\
\text { ginaceae }\end{array}$ & Herb & Lvs,, frt & $\sqrt{ }$ & & & & & General debility, impotency, nerve tonic \\
\hline $\begin{array}{l}75 . \\
\text { Oxalid }\end{array}$ & $\begin{array}{l}\text { Boerhavia rapens } \\
\text { laceae }\end{array}$ & Herb & Ap & $\sqrt{ }$ & & & & & Asthma, diuretic, laxative \\
\hline 76. & Oxalis corniculata & Herb & $\mathrm{Wp}$ & $\sqrt{ }$ & & & & $\sqrt{ }$ & $\begin{array}{l}\text { Skin disease, anti-dysentery and increase } \\
\text { appetite }\end{array}$ \\
\hline \multicolumn{10}{|c|}{ Papaveraceae } \\
\hline 77. & Argemone maxicana & Herb & Wp & & & & $\sqrt{ }$ & & Malarial fever, ulcer, skin problem \\
\hline \multicolumn{10}{|c|}{ Poaceae } \\
\hline 78. & Aeluropus lagopoides & Herb & Wp & $\sqrt{ }$ & & & & & Wound healing, pain killer \\
\hline 79. & Aristida funiculata & Herb & Ap & $\sqrt{ }$ & & & & & Itching and skin problems \\
\hline 80. & Brachiaria reptens & Herb & Wp & $\sqrt{ }$ & & & & & Diuretic, kidney problems \\
\hline 81. & Cenchrus biflorus & Herb & Sds & $\sqrt{ }$ & & & & & Diuretic \\
\hline 82. & Cenchrus ciliaris & Herb & $\mathrm{Wp}$ & $\sqrt{ }$ & & & & & $\begin{array}{l}\text { Lactagogue, kidney pains, tumors, sores and } \\
\text { wounds }\end{array}$ \\
\hline 83. & Cenchrus setigerus & Herb & Ap & $\sqrt{ }$ & & & & & Oxidative stress \\
\hline 84. & $\begin{array}{l}\text { Cymbopogon } \\
\text { jwarancusa }\end{array}$ & Herb & $\mathrm{Wp}$ & $\sqrt{ }$ & & $\sqrt{ }$ & $\sqrt{ }$ & & $\begin{array}{l}\text { Headache, respiratory infections, stomachache, } \\
\text { abdominal and muscle pain, fever }\end{array}$ \\
\hline 85. & Cynodon dactylon & Herb & Ap & $\sqrt{ }$ & $\sqrt{ }$ & & $\sqrt{ }$ & & $\begin{array}{l}\text { Vomiting, leprosy, scabies, dysentery, blood } \\
\text { and skin diseases }\end{array}$ \\
\hline 86. & $\begin{array}{l}\text { Dactyloctenium } \\
\text { aegyptium }\end{array}$ & Herb & Ap & $\sqrt{ }$ & & & & & $\begin{array}{l}\text { Astringent, bitter tonic, intestinal and urinary } \\
\text { diseases }\end{array}$ \\
\hline 87. & Desmostachya bipinnata & Herb & Ap & $\sqrt{ }$ & & & & & Dysentery, diuretic \\
\hline 88. & Dicanthium annulatum & Herb & Ap & $\sqrt{ }$ & & & & & $\begin{array}{l}\text { Dysentery, menorrhagia, bacterial and fungal } \\
\text { infections }\end{array}$ \\
\hline 89. & Eleusine indica & Herb & $\mathrm{Wp}$ & $\sqrt{ }$ & & & & & Liver and kidney problems, oxidative stress \\
\hline 90. & Lasiurus scindicus & & Ap & $\sqrt{ }$ & $\sqrt{ }$ & & & & Cold and cough, bacterial infections \\
\hline 91. & Panicum antidotale & Herb & Sds & $\sqrt{ }$ & $\sqrt{ }$ & & & & Treatment of bone fracture \\
\hline 92. & Phalaris minor & Herb & Ap & $\sqrt{ }$ & & & & & Cough and dysentery \\
\hline
\end{tabular}




\begin{tabular}{|c|c|c|c|c|c|c|c|c|c|}
\hline S. No. & Botanical name & $\begin{array}{l}\text { Growth } \\
\text { Form }\end{array}$ & $\begin{array}{l}\text { Parts } \\
\text { Used }\end{array}$ & Fd & Fw & $\begin{array}{l}\mathbf{F \&} \\
\mathbf{H}\end{array}$ & Orn. & $\begin{array}{l}\text { Fr. \& } \\
\text { Veg. }\end{array}$ & Medicinal Uses \\
\hline 93. & Роа аппиа & Herb & Lvs & $\sqrt{ }$ & & & & & $\begin{array}{l}\text { Kidney and liver ailments, diuretic, digestive } \\
\text { disorders }\end{array}$ \\
\hline 94. & Saccharum bengalensis & Shrub & Ap & $\sqrt{ }$ & & $\sqrt{ }$ & $\sqrt{ }$ & & Diuretic and demulcent \\
\hline 95. & Saccharum spontaneum & Shrub & $\mathrm{Wp}$ & $\sqrt{ }$ & & & & & $\begin{array}{l}\text { Astringent, diuretic, burning sensation piles, } \\
\text { gynecological troubles, respiratory problems }\end{array}$ \\
\hline 96. & Stipagrostis plumosa & Herb & Ap & $\sqrt{ }$ & & & & & Cold and cough \\
\hline 97. & Sorghum halepense & Herb & Ap & $\sqrt{ }$ & $\sqrt{ }$ & & & & Demulcent, diuretic \\
\hline 98. & Vetiveria zizaniodes & Herb & $\mathrm{Wp}$ & $\sqrt{ }$ & $\sqrt{ }$ & $\sqrt{ }$ & & & $\begin{array}{l}\text { Skin disorders, indigestion, tonic and blood } \\
\text { purifier }\end{array}$ \\
\hline \multicolumn{10}{|c|}{ Polygonaceae } \\
\hline 99. & $\begin{array}{l}\text { Calligonum } \\
\text { polygonoides }\end{array}$ & Shrub & $\mathrm{Wp}$ & $\sqrt{ }$ & $\sqrt{ }$ & $\sqrt{ }$ & & & $\begin{array}{l}\text { Typhoid, urinary problems, heart burn and sun } \\
\text { stroke }\end{array}$ \\
\hline 100. & Polygonum plebeium & Herb & $\mathrm{Wp}$ & $\sqrt{ }$ & & & $\sqrt{ }$ & $\sqrt{ }$ & Asthma, cough and pneumonia \\
\hline 101. & Rumex dentatus & Herb & Lvs & $\sqrt{ }$ & & & & $\sqrt{ }$ & Constipation, diuretic, sooth irritation \\
\hline 102. & Rumex crispus & Herb & $\mathrm{Wp}$ & & & & & $\sqrt{ }$ & Cutaneous disorder, viral infections \\
\hline \multicolumn{10}{|c|}{ Portulacaceae } \\
\hline 103. & Portulaca oleracea & Herb & Lvs & & & & & $\sqrt{ }$ & Diabetes, urinary bleeding \\
\hline 104. & Portulaca quadrifida & Herb & Ap & $\sqrt{ }$ & & & & & Fever, diuretic, rheumatism \\
\hline \multicolumn{10}{|c|}{ Primulaceae } \\
\hline 105. & Anagallis arvensis & Herb & $\mathrm{Wp}$ & $\sqrt{ }$ & $\sqrt{ }$ & $\sqrt{ }$ & $\sqrt{ }$ & & $\begin{array}{l}\text { Diuretic, expectorant, emollient, purgative and } \\
\text { skin infections }\end{array}$ \\
\hline \multicolumn{10}{|c|}{ Ranunculaceae } \\
\hline 106. & Ranunculus muricatus & Herb & Ap & $\sqrt{ }$ & $\sqrt{ }$ & & & & Intermittent fevers, gout, asthma \\
\hline 107. & Ranunculus sceleratus & Herb & $\mathrm{Wp}$ & $\sqrt{ }$ & & & & & $\begin{array}{l}\text { Cold, general debility, rheumatism, stomach } \\
\text { problems }\end{array}$ \\
\hline \multicolumn{10}{|c|}{ Solanaceae } \\
\hline 108. & Datura stramonium & Shrub & Sds, Lvs & $\sqrt{ }$ & & & $\sqrt{ }$ & & Earache, asthma, coma and hair fall \\
\hline 109. & Solanum nigrum & Herb & Lvs, fr & $\sqrt{ }$ & & & & $\sqrt{ }$ & Fever, wound healing, stomach disease \\
\hline 110. & Solanum surattense & Herb & $\mathrm{Wp}$ & $\sqrt{ }$ & & & & $\sqrt{ }$ & Jaundice, cough and asthma \\
\hline 111. & Withania coagulens & Herb & Lvs, Fr & $\sqrt{ }$ & & & & $\sqrt{ }$ & Anorexia, jaundice, skin problem \\
\hline $\begin{array}{l}112 . \\
\text { Tamat }\end{array}$ & $\begin{array}{l}\text { Withania somnifera } \\
\text { rcaceae }\end{array}$ & Shrub & $\mathrm{Wp}$ & $\sqrt{ }$ & & & $\sqrt{ }$ & $\sqrt{ }$ & Diuretic and swelling \\
\hline $\begin{array}{l}\text { TamaI } \\
113\end{array}$ & $\begin{array}{l}\text { Tamaricae } \\
\text { Tamaix dioica }\end{array}$ & Shrub & Stm, fr & $\sqrt{ }$ & $\sqrt{ }$ & & & $\sqrt{ }$ & $\begin{array}{l}\text { Cough, diarrhea, dysentery, piles, ulcer, spleen } \\
\text { trouble }\end{array}$ \\
\hline \multicolumn{10}{|c|}{ Verbenaceae } \\
\hline 114. & Lantana camara & Shrub & Lvs, fr & & $\sqrt{ }$ & $\sqrt{ }$ & $\sqrt{ }$ & & Stomach disorders, microbial infections \\
\hline \multicolumn{10}{|c|}{ Zygophyllaceae } \\
\hline 116. & Fagonia cretica & Herb & $\mathrm{Wp}$ & $\sqrt{ }$ & $\sqrt{ }$ & & & & $\begin{array}{l}\text { Fever, dysentery, asthma, liver trouble, } \\
\text { toothache, small pox, skin diseases, typhoid, } \\
\text { urinary discharges }\end{array}$ \\
\hline 117. & Peganum harmala & Herb & Sds, rts & $\sqrt{ }$ & & & $\sqrt{ }$ & & Asthma, fever, antiseptic, skin inflammations \\
\hline 118. & Tribulus terrestris & Herb & $\mathrm{Wp}$ & & & & $\sqrt{ }$ & & Urinary disorder, kidney disorder \\
\hline
\end{tabular}

Fd: Fodder; Fw: Fuel wood; F \& H: Fencing and hedges; Orn.: Ornamental; Fr. \& Veg.: Fruits and vegetables; Ap: aerial parts; Wp: Whole plant; Lvs: Leaves; Fr.: Fruit: Sds: Seeds; Rts: Roots; Stm: Stem; Flw: Flowers.

Most of the collected plant species were found to be used as fodder for domestic animals (96 species) followed by 44 species used as fruit and vegetable. Other uses of plants by local inhabitants include ornamental (33 species), fuel wood for burning (24 species) and fencing \& hedges (11 species) (Fig. 1). Herbs and shrubs were also observed to be used in herbal medicines to cure various diseases such as A. indicum is used to purify the blood, cure cough, asthma and chest infection and can be used as laxative, emollient and demulcent. Similarly, $H$. recurvum is used to cure intestinal ulcers and $C$. flat is used to cure skin diseases. People in different areas use different plant parts according to their knowledge transferred from their ancestors. The most important medicinal uses of plants were related to fever and cold (35 species such as $S$. plumosa) followed by urinary diseases (32 species such as $T$. terrestris), respiratory diseases (25 species such as $S$. spontaneum), skin diseases and diarrhea and constipation (20 species such as $F$. indica and $V$. sativa), digestive disorders (18 species such as $L$. camara), kidney disorders (12 species such as B. reptens), microbial infections (10 species such as $C$. cretica), liver disorders ( 9 species such as $C$. occidentalis), rheumatism and laxative (8 species such 
as $C$. burhia), headache (6 species such as A. aspera), diabetes (5 species such as $C$. didymus), intestinal ulcers (4 species such as $T$. triquetra), general debility, eye disease and small pox and measles (3 species such as $S$. fruticosa) and hair growth and toothache/bleeding gums (2 species such as $E$. alba) respectively (Figure 2). Other medicinal uses include hepatitis, typhoid, jaundice, malaria, tuberculosis, anorexia, piles, dysentery, tumors and inflammations of different body parts. Results revealed that some species are used for various health problems like Fagonia cretica and Calligonum polygonoides were used for multiple diseases (Table 1).
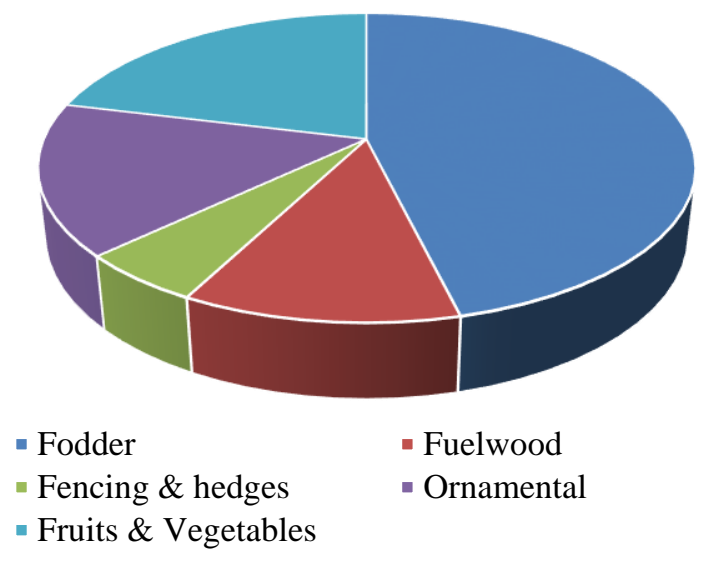

Figure 1. Comparative number of plants used for various purposes by local inhabitants of Tehsil Yazman, Pakistan.

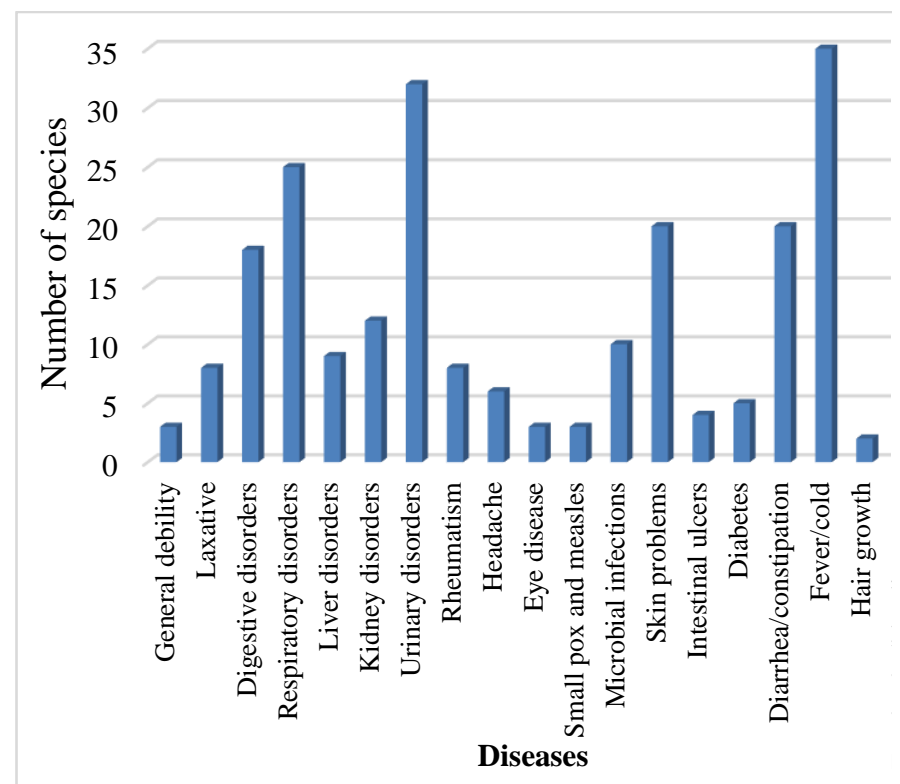

Figure 2. Distribution of medicinal plants with respect to their use in medicine for different diseases in Tehsil Yazman, Pakistan.

\section{DISCUSSION}

Man has been depending upon plants for his survival since ancient time. Both wild and cultivated plants are vital to many aspects of traditional life (Salinitro et al., 2017). The present study was carried out to assess the ethno-medicinal knowledge about the species of Tehsil Yazman with general information and their folk uses. Tehsil Yazman has a great diversity of vegetation and people living there depend upon plants for their needs. Most of the nomads in the area especially cut the trees and sell them as fuelwood. They also use the plants for many other purposes that include thatching (craft of building a roof with dry vegetation) and as ornamental etc. Most of the people have domestic animals that feed on these herbs and shrubs and also use different plants to cure various diseases of animals. Due to poverty and lack of modern health facilities, most people still use traditional medicines for their common day diseases.

Many plants are used as traditional herbs or foods in many countries since ancient times (Wu et al., 2015). In present study, total 118 species belonging to 35 families have been documented. Most of the species were reported to have multipurpose uses by the inhabitants of the respective areas e.g., Sesbania sesban, Calotropis procera and Aloe barbadensis. Similar studies have been done in different areas of Pakistan. Sardar and Khan (2009) conducted ethnobotanical studies of Tehsil Shakargarh, District Narowal, Pakistan and recorded traditional uses of 102 species belonging to 62 families. Mahmood and Shah (2012) conducted survey of medicinal plants in Poonch, District of Jammu and Kashmir and reported total of 65 species in use to cure various diseases. Similarly, Ahmad et al. (2014) conducted ethnobotanical study of Chail valley of District Swat, Pakistan and documented 50 plant species belonging to 35 families. Moreover, Yaseen et al. (2015) also conducted ethnobotanical studies of medicinal plants in the Thar Desert of Pakistan and reported medicinal uses of 87 plant species belonging to 32 families respectively.

People prefer local herbs and shrubs due to low cost, easy availability and more effectiveness to cure different diseases and disorders. According to the 'Hakeems' and 'Pansaries', there are many threats to medicinal plants due to over exploitation and over consumption (Amjad and Arshad, 2014). Present study revealed that Tehsil Yazman possess great diversity of plants that meet the needs of inhabitants. There is no ethnobotanical/ medicinal data available from this remote area. This study contributes to the establishment of an inventory of plant based medicines used in Tehsil Yazman, Pakistan. The data analyzed in this paper show that indigenous knowledge on medicinal plants uses is still alive in this area. The significance of this knowledge has provided us with novel information that can provide basis for new avenues in future pharmacological screening that leads to natural drugs discovery development to improve healthcare 
systems all over the world. However, to validate such information, detailed pharmacological studies must be done to improve the use of medicinal plants. Overall, the present study provided a baseline study for the conservation of the local species. Hence, major attention is required to conserve these wild species for future utilization and to retain the natural glory.

Conclusion: In present studies, the natural flora of Tehsil Yazman has been explored. This area is embraced with ample herbs and shrubs notably utilized by the local people for various purposes including fuelwood, fruit and vegetable, forage/fodder and ornamental. These species are also used for treating various ailments such as stomach, liver and kidney disorders, eye diseases, microbial infections, headache, small pox and measles, fever and cold etc. Hence, this information can be used further to determine the biological potential of species so that they can be effectively employed in pharmaceutical industries. Moreover, present studies can be used for the conservation purposes to protect the valuable flora from biotic stress for future generation.

\section{REFERENCES}

Ahmad, M., S. Sultana, S. Fazl-i-Hadi, Taibi ben Hadda, S. Rashid, M. Zafar, M.A. Khan, M.P. Z. Khan and G. Yaseen. 2014. An ethnobotanical study of medicinal plants in high mountainous region of Chail valley (District Swat, Pakistan). J. Ethnobiol. Ethnomed. 10:36.

Ahmad, K.S., R. Qureshi, M. Hameed, F. Ahmad and T. Nawaz. 2012. Conservation assessment and medicinal importance of some plants resources from Sharda, Neelum Valley, Azad Jammu and Kashmir, Pakistan. Int. J. Agric. Biol. 14:997-1000.

Ahmad, K.S., W.K. Kayani, M. Hameed, F. Ahmad and T. Nawaz. 2012. Floristic diversity and ethnobotany of Sensha, district Kotli, Azad Jammu and Kashmir, Pakistan. Pak. J. Bot. 44:195-201.

Ajaib, M., Z. Khan, N. Khan and M. Wahab. 2010. Ethnomedicinal studies on useful shrubs of district Kotli, Azad Jammu and Kashmir, Pakistan. Pak. J. Bot. 42:14071415.

Ali, S.I. and M. Qaiser. 1986. A phytogeographic analysis of the phanerogams of Pakistan and Kashmir. Proc. Roy. Soc. Edinb. 89:89-101.

Amjad, M.S. and M. Arshad. 2014. Ethnobotanical inventory and medicinal uses of some important woody plants species of Kotli, Azad Kashmir, Pakistan. Asian Pac. J. Trop. Biomed. 4:952-958.

Butt, M.A., M. Ahmad, A. Fatima, S. Sultana, M. Zafar, G. Yaseen, M.A. Ashraf, Z.K. Shinwari and S. Kayani. 2015. Ethnomedicinal uses of plants for the treatment of snake and scorpion bite in Northern Pakistan. J. Ethnopharm. 168:164-181.

Choudhary, K. and K.M. Nama. 2014. Phyto-diversity of Mukundara hills national park of Kota district, Rajasthan, India. Adv. Appl. Sci. Res. 5:18-23.

Mahmood, T. and A. Shah. 2012. Medicinal plants used by traditional healers in Poonch district of Jammu and Kashmir. Life Sci. Leafl. 5:53-60.

Malik, B., R. Urooj, M. Nawaz, S.S. Ahmad and A. Wahid. 2013. An appraisal of ecological distribution of herbaceous flora at Gatwala Forest Park Faisalabad, Pakistan. Middle-East J. Sci. Res. 17:593-599.

Nasir, E. and S.I. Ali. 2001. Flora of Pakistan. National Herbarium, Pakistan; p.200.

Qureshi, R., G.R. Bhatti and G. Shabbir. 2011. Floristic inventory of Pir Mehr Ali Shah Arid Agriculture University Research Farm at Koont and its surrounding areas. Pak. J. Bot. 43:1679-1684.

Salinitro, M., R. Vicentini, C. Bonomi and A. Tassoni. 2017. Traditional knowledge on wild and cultivated plants in the Kilombero Valley (Morogoro Region, Tanzania). J. Ethnobiol. Ethnomed. 13:17.

Sardar, A.A. and Z. Khan. 2009. Ethnomedicinal studies on plant resources of tehsil Shakargarh, District Narowal, Pakistan. Pak. J. Bot. 41:11-18.

Shinwari, S., R. Qureshi and I. Bayound. 2011. Ethnobotanical study of Kohat pass, Pakistan. Pak. J. Bot. 43:135-139.

Wariss, H.M., S.A. Pirzada, K. Alam, S. Anjum and R. Qureshi. 2014. Flora of Lal Suhanra National Park, Bahawalpur, Pakistan. Pak. J. Bot. 46:1331-1341.

Wu, S., T. Xu and D. Huang. 2015. Chemical compositions of the wild extracts from seeds of Dendranthema nankingense and Borago offiinalis. J. Food Drug Anal. 23:253-259.

Yaseen, G., M. Ahmad, S. Sultana, A.S. Alharrasi, J. Hussain, M. Zafar and S. Rehman. 2015. Ethnobotany of medicinal plants in the Thar Desert (Sindh) of Pakistan. J. Ethnopharm. 163:43-59.

Zereen, A. and Z. Khan. 2012. A survey of ethnobotanically important trees of Central Punjab, Pakistan. Biologia 58:21-30. 